\title{
Implementation of Dynamic Bayesian Decision Making by Intracellular Kinetics
}

\author{
Tetsuya J. Kobayashi* \\ Institute of Industrial Science, The University of Tokyo, 4-6-1 Komaba Meguro-ku, Tokyo 153-8505, Japan
}

(Received 20 January 2010; published 3 June 2010)

\begin{abstract}
Decision making in a noisy and dynamically changing environment is a fundamental task for a cell. To choose appropriate decisions over time, a cell must be equipped with intracellular kinetics that can conduct dynamic and efficient decision making. By using the theory of sequential inference, I demonstrate that dynamic Bayesian decision making can be implemented by an intracellular kinetics with a dual positive feedback structure. I also show that the combination of linear instantaneous and nonlinear stationary sensitivities to the input dominantly contributes to decision making efficiency, and that the state-dependent sensitivity change further suppresses noisy response. The statistical principles underlying these two factors are further clarified to be a log-likelihood-dependent quantification of the input information and uncertainty-dependent sensitivity control.
\end{abstract}

DOI: 10.1103/PhysRevLett.104.228104

Cells are subject to unpredictable environmental changes with regard to the presence of nutrients, toxic molecules, or signaling molecules from other cells. While cells respond passively to such an uncertain environment in some cases, they have developed, in other cases, intracellular kinetics that allow them to actively make a decision by quickly detecting a change in the received signals. Depending on the type of decision, the problem of decision making by a cell is relevant to a variety of intracellular phenomena such as differentiation, metabolic switching, apoptosis, and chemotaxis [1-3]. Of particular interest is that cells can make such a decision even with substantial noise in the environment and intracellular reactions [4-8]. However, little is known about what kind of intracellular kinetics can conduct efficient decision making from noisy information.

To address this problem, Bayes theory and rate distortion theory have been applied for chemotaxis, gene regulatory machinery, and development [9-13]. However, only stationary decision making problems have been mainly addressed. The stationary decision making is not sufficient because it requires a versatile memory, which a cell lacks, to store all input, and because a cell makes decisions over time. Yet to be revealed is, therefore, the relation between decision making dynamics and its implementation by intracellular kinetics $[14,15]$. To this end, I formulate the dynamic decision making of a cell as a sequential Bayesian inference problem in which the state of the environment, hidden information for a cell, is estimated from a series of noisy observations.

The simplest decision that a cell makes is whether the environment is in a certain state or another, denoted as the $O$ state and $\emptyset$ state, respectively [Fig. 1(a)]. This random change of the environmental state $x_{t} \in \mathcal{X} \equiv\{\varnothing, O\}$ is statistically modeled by a two-state continuous Markov process in which the transition rate from $x_{t}=\emptyset$ to $x_{t}=O$ and from $x_{t}=O$ to $x_{t}=\emptyset$ is $r_{O}$ and $r_{\varnothing}$, respectively [16]. A
PACS numbers: 87.10.Vg, 87.10.Mn, 87.18.Mp, 87.18.Tt

cell, in general, obtains the information of the state from noisy reactions of receptors on its membrane. Since the state of each receptor is also binary (i.e., active or inactive), the state of receptors can be represented by a vector of binary variables $s_{t}$, where the $j$ th component of $s_{t}$ is the state of the $j$ th receptor at time $t: s_{t}^{j} \in \mathcal{S} \equiv\{0,1\}$ [Fig. 1(a)]. The length of the vector, $N_{0}$, is the total number of recep-
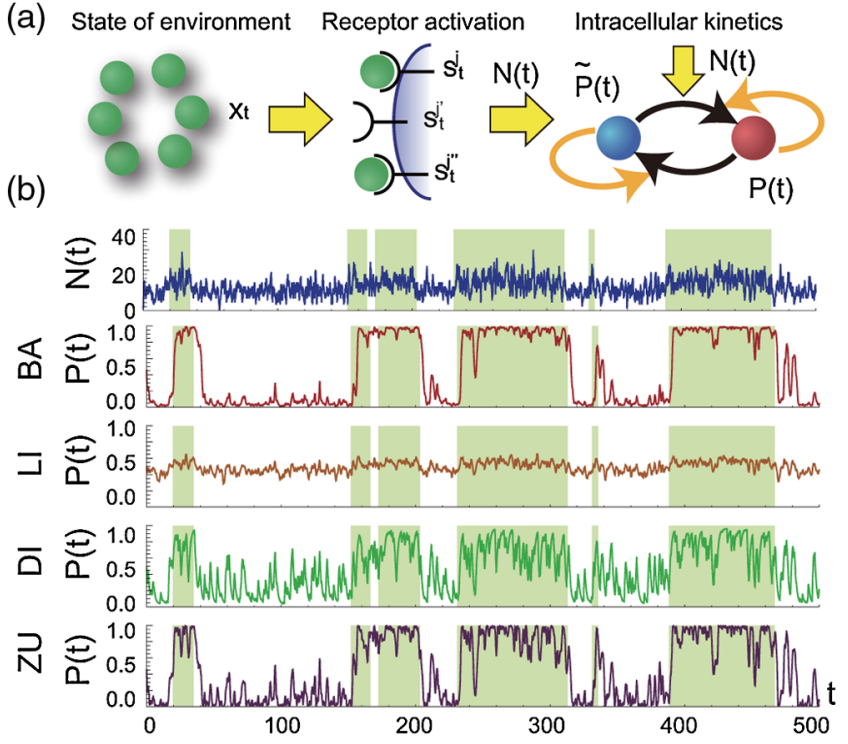

FIG. 1 (color online). (a) Schematic diagram of dynamic decision making of a cell. (b) A sample path of noisy receptor activation, $N(t)$, and the responses of Bayesian (BA), linear input (LI), dual input (DI), and zero-order ultrasensitivity (ZU) kinetics to $N(t)$. The intervals during which $x_{t}=O$ are designated with filled regions. Parameter values are $N_{0}=100, \lambda_{O}=0.14$, $\lambda_{\not \emptyset}=0.1, \quad r_{O}=r_{\not \emptyset}=0.03, \quad \tau=1, \quad v_{\mathrm{LI}}=1 / 6, \quad v_{\mathrm{DI}}=\lambda_{d} / 4$, $n_{\mathrm{DI}}=\left[\left(r_{O}+r_{\varnothing}\right) \lambda_{d}+N_{0} v_{\mathrm{DI}} \lambda_{d}\right] /\left(5 r_{O} v_{\mathrm{DI}}\right), \quad K_{\mathrm{DI}}=\lambda_{d} / \lambda_{r}$, $v_{\mathrm{ZU}}=1 / 4, \quad K_{\text {on }}=K_{\text {off }}=\left(r_{O}+r_{\emptyset}\right) /\left[2\left(N_{0} \lambda_{d}-\left(r_{O}+r_{\varnothing}\right)\right]\right.$. These values are determined so that each kinetics has similar stationary sensitivity and time scale to those of BA kinetics. 
tors. I model the stochastic activation of the $j$ th receptor by a Poisson point process in which the parameter $\lambda^{j}(t)=$ $\lambda^{j}\left(x_{t}\right) \geq 0$ is a function of the state of the environment. For readability, I abbreviate $\lambda^{j}(x=O)$ and $\lambda^{j}(x=\emptyset)$ as $\lambda_{O}^{j}$ and $\lambda_{\phi}^{j}$, respectively.

Next, I derive the statistically optimal kinetics to sequentially infer the posterior probability $\mathbb{P}\left(x_{t} \mid s_{0: t}\right)$ that the environment is $x_{t}$ from the sequence of receptor activities $s_{0: t} . s_{0: t^{\prime}}$ represents the receptor state $s_{t}$ from $t=$ 0 to $t=t^{\prime}$. By using the theory of sequential Bayesian inference [17], I obtain $\mathbb{P}\left(x_{t^{\prime}} \mid s_{0: t^{\prime}}\right)=\mathbb{P}\left(s_{t^{\prime}} \mid x_{t^{\prime}}\right) \times$ $\left[\left(\sum_{x_{t} \in X} \mathbb{P}\left(x_{t^{\prime}} \mid x_{t}\right) \mathbb{P}\left(x_{t} \mid s_{0: t}\right)\right) /\left(\mathbb{P}\left(s_{t^{\prime}} \mid s_{0: t}\right)\right)\right]$, where $t^{\prime}=$ $t+\Delta t$. For sufficiently small $\Delta t$ during which each receptor gets active at most once and the state of the environment changes at most once, I can then obtain the following approximations [18]: $\mathbb{P}\left(s_{t^{\prime}} \mid x_{t^{\prime}}=x\right) \approx \prod_{j}\left[\left(\lambda_{x}^{j} \Delta t\right) /\right.$ $\left.\left(1-\lambda_{x}^{j} \Delta t\right)\right]_{t^{\prime}}^{s^{\prime}}\left(1-\lambda_{x}^{j} \Delta t\right), \mathbb{P}\left(x_{t^{\prime}}=\tilde{x} \mid x_{t}=x\right) \approx r_{\tilde{x}} \Delta t$, and $\mathbb{P}\left(x_{t^{\prime}}=x \mid x_{t}=x\right) \approx\left(1-r_{\tilde{x}} \Delta t\right)$ where $\tilde{x}=\mathcal{X} \backslash x$. For simplicity, I also assume that $\lambda_{x}^{j}=\lambda_{x}$ for all $j$, and $\lambda_{O}^{j}>\lambda_{\varnothing}^{j}$, without loss of generality. An activated receptor is also assumed to get inactive in $\tau$, which is much smaller than $1 / \lambda_{O}$, that is, the average waiting time between consecutive receptor activations. By taking the limit of $\Delta t \rightarrow 0$ under these approximations and assumptions (see supplementary material in Ref. [19] for more detail), I obtain

$$
\frac{d \mathcal{P}(t)}{d t}=\mathcal{P}(t) \tilde{\mathcal{P}}(t)\left[\lambda_{r} N_{0} S(t)-N_{0} \lambda_{d}\right]+r_{O} \tilde{\mathcal{P}}(t)-r_{\phi} P(t),
$$

where $\mathcal{P}(t)$ is an abbreviation of $\mathbb{P}\left(x_{t}=O \mid s_{0: t}\right), \tilde{\mathcal{P}}(t)=$ $1-\mathcal{P}(t), \lambda_{r}=\log \frac{\lambda_{O}}{\lambda_{\phi}}, \lambda_{d}=\lambda_{O}-\lambda_{\phi}, N(t)$ is the total number of active receptors at $t$, and $S(t):=N(t) / N_{0} \tau$ is the effective input per receptor. This kinetics is referred to as Bayesian kinetics (BA kinetics) in this Letter. As shown in Fig. 1(b), the BA kinetics robustly and efficiently identifies changes in the environment even though the input signal $N(t)$ is extremely noisy. This property is not sensitive to a change in $\tau$ when $\tau$ is not very large.

The dynamics of this equation can be identified with a covalent modification reaction with autoregulatory feedbacks [Fig. 1(a)] if the equation is rearranged as follows:

$$
\frac{d \mathcal{P}}{d t}=F_{\mathrm{BA}}(\mathcal{P}, S(t))-G_{\mathrm{BA}}(\mathcal{P}, S(t))+r_{O} \tilde{\mathcal{P}}-r_{\varnothing} \mathcal{P},
$$

where $F_{\mathrm{BA}}(\mathcal{P}, S(t))$ and $G_{\mathrm{BA}}(\mathcal{P}, S(t))$ are defined in Table I. In Eq. (1), $\mathcal{P}$ corresponds to the ratio of molecules in the active state in the covalent modification reaction [Fig. 1(a)]. $F_{\mathrm{BA}}(\mathcal{P}, S(t))$ can be regarded as an activation reaction that is induced by active receptors and is also positively regulated by activated molecules as autophosphorylation does. In contrast, $G_{\mathrm{BA}}(\mathcal{P}, S(t))$ is a spontaneous inactivation reaction, which is positively regulated by inactive molecules. Furthermore, $r_{O} \tilde{\mathcal{P}}(t)$ and $r_{\varnothing} \mathcal{P}(t)$ can be interpreted as leaky activation and inactivation without the autoregulations, respectively. Depending on the parameter values of the autoregulatory and leaky reactions, Eq. (1) can be interpreted as a covalent modification reaction with either dual positive feedbacks, single positive feedback, or no feedback. Such a modification reaction with a single positive feedback loop is a typical motif of the signaling network for chemotaxis, for example, which consists of the reaction of PIP2 and PIP3 via Cdc42/Ras [20]. This motif enables a chemotactic cell to robustly determines the direction of ligand sources even though the directional information is substantially impaired by noisy reactions [6,10,21,22]. Moreover, autophosphorylative regulation has been observed in various important intracellular kinetics [23]. In addition, dual positive feedback structures have recently been observed experimentally in the gene regulatory networks for the determination of the fates of embryonic stem cells and $T$ cells $[1,24,25]$. These facts suggest that statistically efficient kinetics may be employed in intracellular reactions to conduct decision making and signal processing.

In order to further investigate the efficiency of BA kinetics, I analyzed three different intracellular kinetics: linear input kinetics (LI kinetics), dual input kinetics (DI kinetics), and zero-order ultrasensitivity kinetics (ZU kinetics) [26]. These kinetics are obtained by modifying the autoregulatory activation and inactivation reactions of BA kinetics as summarized in Table I. Figure 1(b) shows the responses of these kinetics to the same noisy input as that of BA kinetics. Of the three kinetics, the simplest, LI kinetics, which lacks autoregulatory interactions, cannot respond sharply to the input signal [Fig. 1(b)], which is quantified by the highest error rate (ER) and mean absolute error (MAE) in Figs. 2(a) and 2(b) at $\lambda_{O}=0.14$. As demonstrated in Fig. 3, the stationary response of LI kinetics to constant input $S$ is a Michaelis-Menten type, while the other kinetics show highly nonlinear sigmoidal responses. This result indicates that high stationary sensitivity is required for efficient decision making. However, the stationary sensitivity is not sufficient for the efficiency because the ER and MAE of DI kinetics are much higher than those of ZU and BA kinetics (Fig. 2).

As obviously seen from Table I, the dominant difference between DI kinetics and $\mathrm{ZU}$ and BA kinetics is the origin

\begin{tabular}{|c|c|c|c|c|}
\hline$z$ & LI & DI & $\mathrm{ZU}$ & BA \\
\hline$F_{z}(\mathcal{P}, S)$ & {$\left[v_{\mathrm{LI}} N_{0} \lambda_{r} S\right] \tilde{\mathcal{P}}$} & {$\left[v_{\mathrm{DI}} N_{0} \frac{S^{n} \mathrm{DI}}{S^{n} \mathrm{DI}+K_{\mathrm{I}}{ }^{n} \mathrm{DI}}\right] \tilde{\mathcal{P}}$} & {$\left[v_{\mathrm{ZU}} N_{0} \lambda_{r} S\right]_{\frac{\tilde{\mathcal{P}}+K_{0}}{\tilde{\mathcal{P}}}}$} & {$\left[N_{0} \lambda_{r} S \mathcal{P}\right] \tilde{\mathcal{P}}$} \\
\hline$G_{z}(\mathcal{P}, S)$ & {$\left[v_{\mathrm{LI}} N_{0} \lambda_{d}\right] \mathcal{P}$} & {$\left[v_{\mathrm{DI}} N_{0} \frac{K_{\mathrm{DI}}^{n_{\mathrm{DI}}}}{S^{n} \mathrm{DI}+K_{\mathrm{DI}}^{\mathrm{DI}}}\right] \mathcal{P}$} & {$\left[v_{\mathrm{ZU}} N_{0} \lambda_{d}\right] \frac{\mathcal{P}}{\mathcal{P}+K_{\text {off }}}$} & {$\left[N_{0} \lambda_{d} \tilde{\mathcal{P}}\right] \mathcal{P}$} \\
\hline
\end{tabular}

TABLE I. $\quad F_{z}(\mathcal{P}, S)$ and $G_{z}(\mathcal{P}, S)$ for $z=$ linear input (LI), dual input (DI), zero-order ultrasensitivity (ZU), and BA kinetics. 

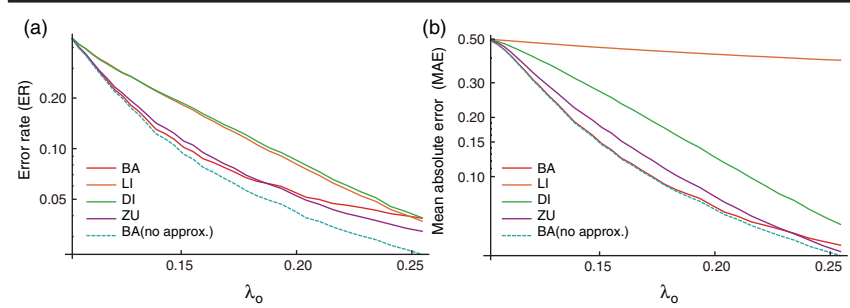

FIG. 2 (color). The error rates defined as $\frac{1}{T} \int_{0}^{T} d t I d\left(\mid x_{t}-\right.$ $\mathcal{P}(t) \mid>\frac{1}{2}$ ) (a), mean absolute error defined as $\frac{1}{T} \times$ $\int_{0}^{T} d t\left|x_{t}-\mathcal{P}(t)\right|(\mathrm{b})$ of each kinetics as a function of $\lambda_{O}$ where $\operatorname{Id}(u)=1$ when $u$ is true and $\operatorname{Id}(u)=0$ otherwise. The red, orange, green, and purple solid curves correspond to BA, LI, DI, and ZU kinetics, respectively. The dashed curve represents the result obtained by BA kinetics with infinitely small $\tau$.

of the nonlinearity in the stationary response. In DI kinetics, the nonlinearity in the stationary response is attributed to the nonlinear dependency of $F_{\mathrm{DI}}$ and $G_{\mathrm{DI}}$ on $S$. In other words, the nonlinear stationary sensitivity comes from the nonlinear instantaneous sensitivity to the input. From the viewpoint of linear response theory, this result is natural because amplification of noise accompanies the high stationary sensitivity that is required for a quick response to a change in the environment [27,28]. The trade-off between a quick response and robustness to noise cannot be resolved except by employing nonstationary dynamics of kinetics. In contrast to DI kinetics, ZU and BA kinetics show nonlinear stationary sensitivity even though their $F(\mathcal{P}, S)$ depend linearly on $S$. This means that their nonlinear stationary sensitivity results from the intrinsic dynamics of these kinetics. Since both ZU and BA kinetics have low ER (Fig. 2), the combination of the nonlinear stationary sensitivity and the linear instantaneous sensitivity is the dominant factor of dynamic decision making by which a cell can quickly detect the actual change of the environment.

Although there is not much difference between ERs of $\mathrm{ZU}$ and BA kinetics, the MAE of BA kinetics is much lower than that of ZU kinetics. This means that BA kinetics is less sensitive to input noise than ZU kinetics when the environment is not changing, while it can detect the change of environment as fast as ZU kinetics. This robustness to input noise can be explained by the state-dependent sensitivity change, which is implemented in BA kinetics as the

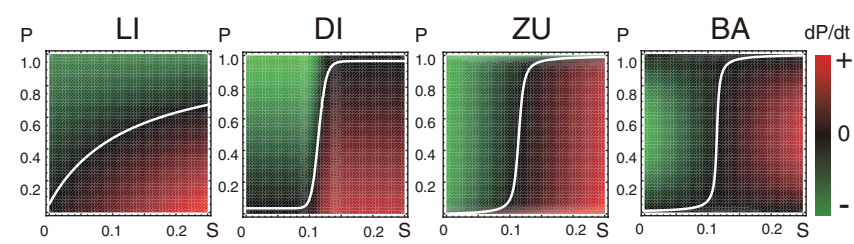

FIG. 3 (color). The phase space diagram of LI, DI, ZU, and BA kinetics as a function of $\mathcal{P}$ and $S . d \mathcal{P} / d t$ is shown by colors. Each white curve is the stationary response of each kinetics to $S$, which satisfies $d \mathcal{P} / d t=0$. quadratic dependence of $F(\mathcal{P}, S)$ on $\mathcal{P}$. Owing to this dependency, $\mathcal{P}(t)$ of BA kinetics changes only when the deviation of the input $S(t)$ from the expected value of the current estimate continues for a certain time, which is induced by the actual change in the state of the environment. In other words, the state-dependent sensitivity makes BA kinetics less sensitive to the input noise by enabling it to optimally integrate the temporally consecutive information of the input. In summary, the efficiency of the decision making dynamics is determined dominantly by the combination of the nonlinear stationary sensitivity and the linear instantaneous sensitivity to $S$, and the quadratic dependence of the instantaneous sensitivity on $\mathcal{P}$. Since these properties can be quantified experimentally, they will be good experimental targets to test whether a given intracellular kinetics can potentially conduct statistically efficient decision making.

The meaning of these factors for the efficiency of dynamic decision making can be further clarified from the statistical viewpoint. Let $\mathbb{P}_{B}(x ; p)$ be a Bernoulli distribution on $x \in \mathcal{X}$ with the success parameter $p$, and let $\mathbb{P}_{P_{o}}(n ; \lambda)$ be a Poisson distribution with the mean parameter $\lambda$. Then, Eq. (1) can be represented as

$$
\frac{d \mathcal{P}(t)}{d t}=V_{B}(\mathcal{P}(t)) I(N(t))+r_{O} \tilde{\mathcal{P}}(t)-r_{\varnothing} \mathcal{P}(t),
$$

where $\quad V_{B}(\mathcal{P})=\mathcal{P} \tilde{\mathcal{P}}, \quad$ and $\quad I(N(t))=$ $\log \mathbb{P}_{P o}\left(N(t) ; \tau N_{0} \lambda_{O}\right)-\log \mathbb{P}_{P o}\left(N(t) ; \tau N_{0} \lambda_{\not}\right) . I(N(t))$ represents the information about the current state of the environment obtained from the input $N(t)$, and the second term of Eq. (2) predicts the future state from the current estimate by using the prior knowledge. The information, $I(N(t))$, is independent of $\mathcal{P}(t)$ and is quantitatively measured by the difference between the log likelihoods that $N(t)$ active receptors are observed in the two environmental states. The linearity of the instantaneous sensitivity is, therefore, inherited from the Poisson statistics of the receptor activations. The information obtained from the receptor activations is subsequently integrated into $\mathcal{P}$ after being weighted with $V_{B}(\mathcal{P}(t))$. Since $V_{B}(\mathcal{P}(t))$ is the variance of $\mathbb{P}_{B}(x ; \mathcal{P})$, it can be interpreted as a measure of the uncertainty in the current estimate of the environment. When the uncertainty of the estimate, $V_{B}$, is large, then the information of the input signal is emphasized. When the uncertainty of the estimate is small, in contrast, the input information is less emphasized, leading to statistically optimal integration of consecutive information from the input. The nonlinear stationary sensitivity automatically accompanies the combination of this kinetics and the linear term when $r_{O}$ is close to $r_{\varnothing}$ and the contribution of the linear term is much smaller than that of $V_{B}(\mathcal{P}(t)) I(N(t))$. Therefore, the statistical principle underlying the high performance of BA kinetics is characterized by the log-likelihood-dependent quantification of input information and the uncertainty-dependent sensitivity control. 
As already demonstrated, the application of statistical inference to intracellular kinetics gives us fruitful new insights into cellular dynamics and its functions. However, the inference theory is not self-contained for characterization of intracellular kinetics because inference corresponds only to the decoding of information from observed input signals. Encoding and transduction of information also play crucial roles for the information processing. In this Letter, for example, the encoding and the transduction processes correspond to the environmental dynamics, $x_{t}$, and the stochastic receptor kinetics, $s_{0: t}$, respectively. Even though the optimal inference kinetics is employed, the accuracy of the inferred state will be poor when $s_{0: t}$ carries little information on $x_{t}$, as shown in the region where $\lambda_{O}<0.12$ in Fig. 2. In contrast, if $s_{0: t}$ carries sufficient information on $x_{t}$ (for example, $\lambda_{O}>0.22$ in Fig. 2), the state of the environment can be inferred (decoded) even with a suboptimal kinetics, and the efficiency of BA kinetics can be lower than others when $\tau$ is not sufficiently small. In the information theory, such information of $x_{t}$ that $s_{0: t}$ contains can be quantified by the mutual information between $x_{t}$ and $s_{0: t}, I\left[x_{t} ; s_{0: t}\right]:=$ $\iint \mathbb{P}\left(x_{t}, s_{0: t}\right) \log \left[\left(\mathbb{P}\left(x_{t}, s_{0: t}\right)\right) /\left(\mathbb{P}\left(x_{t}\right) \mathbb{P}\left(s_{0: t}\right)\right)\right] d x_{t} d s_{0: t} . \quad$ In my case, $I\left[x_{t} ; s_{0: t}\right]$ is explicitly calculated as

$$
I\left[x_{t} ; s_{0: t}\right]=\int D_{\mathrm{KL}}\left(\mathbb{P}_{B}(Z ; \mathcal{P}) \| \mathbb{P}_{B}\left(Z ; \overline{\mathcal{P}}_{O}\right)\right) \rho_{t}(\mathcal{P}) d \mathcal{P} .
$$

$D_{\mathrm{KL}}\left(\mathbb{P} \| \mathbb{P}^{\prime}\right)$ is the Kullback-Leibler divergence between the probability distribution $\mathbb{P}$ and $\mathbb{P}^{\prime}, \overline{\mathcal{P}}_{O}=r_{O} /\left(r_{O}+\right.$ $\left.r_{\varnothing}\right)$, and $\rho_{t}\left(\mathcal{P}^{\prime}\right)=\int \delta\left(\mathcal{P}^{\prime}-\mathbb{P}\left(x_{t}=\left.O\right|_{s_{0: t}}\right)\right) \mathbb{P}\left(s_{0: t}\right) d s_{0: t}$. Since $\mathcal{P}(t)=\mathbb{P}\left(x_{t}=O \mid s_{0: t}\right), \rho_{t}\left(\mathcal{P}^{\prime}\right)$ is the probability that $\mathcal{P}(t)$ becomes $\mathcal{P}^{\prime}$ at $t$ calculated by an infinite number of independent realizations of $x_{0: t}$ and $s_{0: t}$. Because $D_{\mathrm{KL}}\left(\mathcal{P}_{B}(Z ; \mathcal{P}) \| \mathcal{P}_{B}\left(Z ; \overline{\mathcal{P}}_{O}\right)\right)$ is concave with respect to $\mathcal{P}$, the mutual information is high when $\rho_{t}(\mathcal{P})$ consolidates near $\mathcal{P}(t)=0$ or $\mathcal{P}(t)=1$. Because the variance of $\mathcal{P}_{B}(Z ; \mathcal{P})$ is small when $\mathcal{P}(t)$ is close to either 0 or 1 , this condition means that the inferred state of $x_{t}$ has little statistical ambiguity when the mutual information is high. The total performance of the intracellular information processing [Fig. 1(a)] is, therefore, determined by the combination of the efficiencies of information transduction, $x_{0: t} \rightarrow s_{0: t}$, and decoding, $s_{0: t} \rightarrow \mathcal{P}(t)$. Moreover, if the kinetics of $x_{t}$ is biologically controllable as when the input $S$ comes from other cells, an efficient encoding by appropriate design of the kinetics of $x_{t}$ will also be important for the intracellular information processing. Thus, a comprehensive theory for information encoding [12], information transmission $[29,30]$, and information decoding has to be developed for understanding the informationtheoretic aspects of more complicated biological phenomena $[9,12,31,32]$.

I thank Yohei M. Koyama, Yoshiyuki Sato, Yoshihiro Morishita, and Ryo Yokota for fruitful discussions. This work was supported by the JST PRESTO program.
*Also at PREST, Japan Science and Technology Agency (JST), 4-1-8 Honcho Kawaguchi, Saitama 332-0012, Japan. tetsuya@mail.crmind.net; http://research. crmind.net/

[1] B. D. Macarthur, A. Ma'ayan, and I. R. Lemischka, Nat. Rev. Mol. Cell Biol. 10, 672 (2009).

[2] A. Pai and L. You, Mol. Syst. Biol. 5, 286 (2009).

[3] X.-P. Zhang, F. Liu, Z. Cheng, and W. Wang, Proc. Natl. Acad. Sci. U.S.A. 106, 12245 (2009).

[4] V. Shahrezaei and P. S. Swain, Curr. Opin. Biotechnol. 19, 369 (2008).

[5] D. Fraser and M. Kaern, Mol. Microbiol. 71, 1333 (2009).

[6] M. Ueda and T. Shibata, Biophys. J. 93, 11 (2007).

[7] W. J. Blake, M. Kaern, C. R. Cantor, and J. J. Collins, Nature (London) 422, 633 (2003).

[8] W. J. Blake et al., Mol. Cell 24, 853 (2006).

[9] D. Mortimer et al., Proc. Natl. Acad. Sci. U.S.A. 106, 10296 (2009).

[10] B. W. Andrews and P. A. Iglesias, PLoS Comput. Biol. 3, e153 (2007).

[11] E. Libby, T. J. Perkins, and P. S. Swain, Proc. Natl. Acad. Sci. U.S.A. 104, 7151 (2007).

[12] Y. Morishita and Y. Iwasa, Phys. Rev. E 77, 041909 (2008).

[13] T. J. Perkins and P. S. Swain, Mol. Syst. Biol. 5, 326 (2009).

[14] R. Losick and C. Desplan, Science 320, 65 (2008).

[15] T. Helikar, J. Konvalina, J. Heidel, and J. A. Rogers, Proc. Natl. Acad. Sci. U.S.A. 105, 1913 (2008).

[16] C. W. Gardiner, Handbook of Stochastic Methods (Springer, New York, 2004).

[17] J.O. Berger, Statistical Decision Theory and Bayesian Analysis (Springer, New York, 1993).

[18] S. Denève, Neural Comput. 20, 91 (2008).

[19] See supplementary material at http://link.aps.org/ supplemental/10.1103/PhysRevLett.104.228104 for the detail of the derivation of the BA kinetics.

[20] H. Naoki, Y. Sakumura, and S. Ishii, J. Theor. Biol. 255, 259 (2008).

[21] B. W. Andrews, T. M. Yi, and P. A. Iglesias, PLoS Comput. Biol. 2, e154 (2006).

[22] W. J. Rappel and H. Levine, Phys. Rev. Lett. 100, 228101 (2008).

[23] A. Cooper and A. MacAuley, Proc. Natl. Acad. Sci. U.S.A. 85, 4232 (1988).

[24] C. Bergmann, J. L. Van Hemmen, and L. A. Segel, Bull. Math. Biol. 63, 405 (2001).

[25] H. Niwa, Y. Toyooka, D. Shimosato, D. Strumpf, K. Takahashi, R. Yagi, and J. Rossant, Cell 123, 917 (2005).

[26] A. Goldbeter and D. Koshland, Proc. Natl. Acad. Sci. U.S.A. 78, 6840 (1981).

[27] J. Paulsson, Nature (London) 427, 415 (2004).

[28] T. Shibata, Proc. Natl. Acad. Sci. U.S.A. 102, 331 (2005).

[29] G. Tkacik, C. G. Callan, and W. Bialek, Proc. Natl. Acad. Sci. U.S.A. 105, 12265 (2008).

[30] F. Tostevin and P. R. ten Wolde, Phys. Rev. Lett. 102, 218101 (2009).

[31] T. Gregor, D. W. Tank, E. F. Wieschaus, and W. Bialek, Cell 130, 153 (2007).

[32] W.-J. Rappel and H. Levine, Proc. Natl. Acad. Sci. U.S.A. 105, 19270 (2008) 